\title{
Optimized Internal Control and Gene Expression Analysis in Epstein-Barr Virus-Transformed Lymphoblastoid Cell Lines
}

\author{
Hye-Young Nam, Hye-Ryun Kim, Sung-Mi Shim, \\ Jae-Eun Lee, Jun-Woo Kim, Hye-Kyung Park, \\ Bok-Ghee Han* and Jae-Pil Jeon*
}

National Biobank of Korea, Center for Genome Science, Korea National Institute of Health, Korea Centers for Disease Control \& Prevention, Osong Health Technology Administration Complex (OHTAC), Chungbuk 363-951, Korea

\begin{abstract}
The Epstein-Barr virus-transformed lymphoblastoid cell line (LCL) is one of the major genomic resources for human genetics and immunological studies. Use of LCLs is currently extended to pharmacogenetic studies to investigate variations in human gene expression as well as drug responses between individuals. We evaluated four common internal controls for gene expression analysis of selected hematopoietic transcriptional regulatory genes between B cells and LCLs. In this study, the expression pattern analyses showed that TBP (TATA box-binding protein) is a suitable internal control for normalization, whereas GAPDH (glyceraldehyde-3-phosphate dehydrogenase) is not a good internal control for gene expression analyses of hematopoiesis-related genes between B cells and LCLs at different subculture passages. Using the TBP normalizer, we found significant gene expression changes in selected hematopoietic transcriptional regulatory genes (downregulation of RUNX1, RUNX3, CBFB, TLE1, and NOTCH2; upregulation of $M S C$ and PLAGL2) between $\mathrm{B}$ cells and LCLs at different passage numbers. These results suggest that these hematopoietic transcriptional regulatory genes are potential cellular targets of EBV infection, contributing to EBV-mediated B-cell transformation and LCL immortalization.
\end{abstract}

Keywords: lymphoblastoid cell line, internal control, quantitative real-time polymerase chain reaction

*Corresponding authors: E-mail jpjeon@cdc.go.kr

Tel +82-43-719-8911, Fax +82-43-719-8949

E-mail bokghee@nih.go.kr

Tel +82-43-719-8801, Fax +82-43-719-8802

Accepted 30 August 2011

\section{Introduction}

EBV-infected B cells are transformed into continuously proliferating lymphoblastoid cell lines (LCLs), which provide genomic resources for human genetics and immunological studies. However, there is a limitation of LCL utilization, due to possible genetic changes in the process of EBV transformation (Shukla and Dolan, 2005; Haas et al., 2006). It is controversial that LCLs are suitable as an experimental material of genomewide association studies (Simon-Sanchez et al., 2007). Nonetheless, the use of LCLs is currently extended to pharmacogenetic studies to investigate variations in human gene expression as well as drug responses between individuals. For example, gene expression phenotypes were investigated in LCLs for the relative impact of nucleotide and copy number variation (Stranger et al., 2007).

EBV infection induces gene expression changes in Bcells, contributing to immortalization. A number of cellular genes have been identified as targets of EBV infection in various systems, including inducible and stable lymphoma cell lines that have been transfected with particular viral gene expression vectors or infected with EBV (Carter et al., 2002; Kelly et al., 2002; CahirMcFarland et al., 2004; Kang et al., 2005). For gene expression analysis, appropriate internal controls should be carefully selected to normalize the expression of target genes in LCLs. Generally, housekeeping genes are used as internal controls for gene expression studies, assuming that such genes exhibit relatively constant basal levels of gene expression under all conditions. However, not all housekeeping genes are always applied to the normalization of gene expression data under all conditions (Thellin et al., 1999). It has been reported that the comparison of target gene expression needs particular internal controls that are suitable in different tissues or different physiological conditions in human and other organisms (Selvey et al., 2001; Vandesompele et al., 2002; Lossos et al., 2003; Filby and Tyler, 2007; Theis et al., 2007; Rho et al., 2010). Among housekeeping genes, GAPDH has been demonstrated to be a good internal control in tumor cell lines (Janssens et al., 2004), while HPRT has been identified as a good reference gene for cancer research when comparing solid tumor tissue samples with normal tissue samples (de Kok et al., 2005; Ohl et al., 2005). Thus, it is recommended that suitable internal controls should be determined for accu- 
rate normalization of gene expression data.

For peripheral blood cells or leukemia/lymphoma cell lines, GAPDH is frequently used to normalize gene expression data (Natkunam et al., 2005; Saunders et al., 2005). According to our previous microarray data, the choice of $G A P D H$ may be problematic as an internal control of gene expression analysis before and after EBV infection. In addition, as the use of LCLs is expanded to various areas of genomics, including pharamcogenomics, gene expression analysis of LCLs needs optimized internal controls. Here, we evaluated four common internal controls for gene expression analysis between B cells and LCLs, applying them to an expression analysis of selected hematopoietic transcriptional regulatory genes. $T B P$ was finally selected as an optimal internal control for gene expression analysis between B cells and LCLs.

\section{Methods}

\section{Cell culture and sample preparation}

Peripheral blood was obtained from the Jungang Blood Center of the Korean Red Cross. Ficoll-Hypaque gradient centrifugation was performed to isolate peripheral blood mononuclear cells according to the manufacturer's instructions (Amersham). Primary CD19(+) B cells were then purified by negative selection using a $B$ cell isolation kit (Miltenyi Biotech). EBV viral stock was collected from the culture of an EBV-transformed B95-8 marmoset cell line, which was maintained at $37^{\circ} \mathrm{C}$ and $5 \% \mathrm{CO}_{2}$ for $4 \sim 7$ days and then stored at $-80^{\circ} \mathrm{C}$ until needed. EBV infection of primary $B$ cells and LCL generation were performed as described elsewhere (Hur et al., 2005). Briefly, EBV-infected B-cells were incubated in RPMI-1640 supplemented with $10 \%$ FBS and penicillin-streptomycin $(100 \mu \mathrm{g} / 100$ unit) for $10 \sim 20$ days until clumps of EBV-infected $B$ cells were visible. The culture medium was then changed with fresh complete medium for subsequent subculture. When cell numbers of EBV-infected B-cells reached approximately 10 million cells per culture flask, the cells were used for the next passage at a seeding density of $2 \sim 5 \times 10^{5}$ cells/ $\mathrm{ml}$. At this point, the LCL was referred as passage number 1. Next, the LCL culture was divided for continuous subculture with fresh medium approximately twice per week and maintained to passage number 100.

\section{Expression analysis}

Total RNA was isolated from B-cells and counterpart LCLs from three donors using Trizol (Invitrogen) and treated with DNase I prior to the synthesis of first-strand
cDNA using oligo (dT) primer and the Superscript II reverse transcription kit (Invitrogen). Semi-quantitative RTPCR was performed for some selected genes. Gene expression levels of some selected genes were estimated by semiquantitative RT-PCR with the following conditions: 25 cycles of $40 \mathrm{sec}$ at $94^{\circ} \mathrm{C}, 40 \mathrm{sec}$ at $60^{\circ} \mathrm{C}$, and $100 \mathrm{sec}$ at $72^{\circ} \mathrm{C}$ for RUNX3, MSC, and GAPDH and 32 cycles for NOTCH2 and TLE1.

For quantitative real-time PCR, amplification mixtures (50 ul) contained 10 100 ng of first-strand cDNA template, 25 ul of $2 \times$ SYBR Green Master Mix buffer (Invitrogen), and $600 \mathrm{nM}$ of forward and reverse primers (Table 1) for: control genes $\beta$-actin (ACTB), glyceraldehyde-3-phosphate dehydrogenase (GAPDH), $\beta$ 2-microglobulin (B2M), and TATA box-binding protein (TBP); and target genes pleiomorphic adenoma gene-like 2 (PLAGL2), musculin (MSC)/activated B-cell factor (ABF1), transducin-like enhancer of split 1 (TLE1), Notch homolog 2 (NOTCH2), Runt-related transcription factor 1 ( $R U N X 1)$, Runt-related transcription factor 3 ( $R U N X 3)$, and core binding factor beta subunit (CBFB). PCR cycles were $95^{\circ} \mathrm{C}$ for $10 \mathrm{~min}$, followed by 40 cycles of $95^{\circ} \mathrm{C}$ for $15 \mathrm{sec}$, and $60^{\circ} \mathrm{C}$ for $1 \mathrm{~min}$. Reactions were run on an $\mathrm{ABI} H \mathrm{HT} 7900$ (Applied Biosystems). Relative expression levels, $\Delta\left(\mathrm{CT}_{\text {gene- }}-\mathrm{CT}_{\text {control }}\right)$, were displayed as the difference in $\mathrm{C}_{\mathrm{T}}$ (threshold cycle) between genes of interest and internal control genes.

Table 1. Sequence information of PCR primers

\begin{tabular}{|c|c|c|}
\hline Gene & Primer sequence & $\begin{array}{l}\text { Size } \\
\text { (bp) }\end{array}$ \\
\hline \multirow[t]{2}{*}{ ACTB } & Forward 5'-GTCTTCCCCTCCATCGTG-3' & 280 \\
\hline & Reverse 5'-TGATCTGGGTCATCTTCTCG-3' & \\
\hline \multirow[t]{2}{*}{ GAPDH } & Forward 5'-CAGGGCTGCTITAACTCTGGTAA-3' & 99 \\
\hline & Reverse 5'-GTGGAATCATATTGGAACATGTAAACC-3' & \\
\hline \multirow[t]{2}{*}{$B 2 M$} & Forward 5'-TाTCATCCATCCGACATTGA-3' & 228 \\
\hline & Reverse 5'-ССTCCATGATGCTGCTTACA-3' & \\
\hline \multirow[t]{2}{*}{$T B P$} & Forward 5'-TTCGGAGAGTTCTGGGATTGTA-3' & 227 \\
\hline & Reverse 5'-TGGACTGTTCTTCACTCTTGGC-3' & \\
\hline \multirow[t]{2}{*}{ PLAGL2 } & Forward 5'-AGGCAGCTGAGCTTTCAGAG-3' & 191 \\
\hline & Reverse 5'-CTCCCGATTCAGGTCAAAAA-3' & \\
\hline \multirow[t]{2}{*}{ MSC } & Forward 5'-CAACCTGTGTGGGGTCTTCT-3' & 215 \\
\hline & Reverse 5'-TCGCAGGATCACTTGCTATG-3' & \\
\hline \multirow[t]{2}{*}{ NOTCH2 } & Forward 5'-AAGCAGAGTCCCAGTGCCTA-3' & 171 \\
\hline & Reverse 5'-CAGGGGGCACTGACAGTAAT-3' & \\
\hline \multirow[t]{2}{*}{ TLE1 } & Forward 5'-CGACAAGTCCATCAGCAGAA-3' & 150 \\
\hline & Reverse 5'-CCCAGATCACCCAGAAAGAA-3' & \\
\hline \multirow[t]{2}{*}{$R U N X 1$} & Forward 5'-TCTAGCTCAGCACTGCTCCA-3' & 232 \\
\hline & Reverse 5'-TCATGCAAAACTGGCTTCAG-3' & \\
\hline \multirow[t]{2}{*}{$R \cup N X 3$} & Forward 5'-TAGGAAGCACGAGGAAAGGA-3' & 189 \\
\hline & Reverse 5'-GCAAACGATAGTGCAAAGCA-3' & \\
\hline \multirow[t]{2}{*}{$C B F B$} & Forward 5'-GCAGGCAAGGTATATTTGAAGG-3' & 218 \\
\hline & Reverse 5'-TTGGAACCAGGACTAGGGTCT-3 & \\
\hline
\end{tabular}


For the correlation coefficient analysis of gene expression patterns, expression levels of particular genes of interest were normalized to four different reference genes. Then, correlation coefficients were calculated from the expression levels ( $\mathrm{C}_{\mathrm{T}}$ values), normalized to pairs of reference genes (e.g., ACTB vs TBP, ACTB vs
$B 2 M, A C T B$ vs $G A P D H, T B P$ vs $B 2 M, T B P$ vs $G A P D H$, $B 2 M$ vs GAPDH).

\section{Results}

To address the question of whether GAPDH is a suit-

Table 2. Expression levels extracted from our previous microarray data

\begin{tabular}{|c|c|c|c|}
\hline Genes & Accession & Description & Fold $^{\mathrm{a}}$ \\
\hline GAPDH & AA419281 & Glyceraldehyde-3-phosphate dehydrogenase & $18.90 \pm 5.59$ \\
\hline B2M & AA670408 & Beta-2-microglobulin & $1.2 \pm 0.14$ \\
\hline TBP & N50549 & TATA box binding protein & $0.7 \pm 0.09$ \\
\hline$A C T B$ & AW081870 & Beta, actin & $4.13 \pm 0.90$ \\
\hline PLAGL1 & Al346457 & Pleiomorphic adenoma gene-like 2 & $4.95 \pm 1.93$ \\
\hline$M S C$ & AA470081 & Musculin (activated B-cell factor-1) & $3.99 \pm 1.65$ \\
\hline TLE1 & Al268473 & Transducin-like enhancer of split 1 (E(sp1) homolog, Drosophila) & $0.06 \pm 0.03$ \\
\hline$R \cup N X 1$ & Al521317 & Runt-related transcription factor 1 (acute myeloid leukemia 1 ; aml1 oncogene) & $0.27 \pm 0.09$ \\
\hline$R U N X 3$ & N67778 & Runt-related transcription factor 3 & $1.5 \pm 0.05$ \\
\hline$C B F B$ & AA187148 & Core-binding factor, beta subunit & $0.68 \pm 0.03$ \\
\hline NOTCH2 & AA827551 & Notch homolog 2 (Drosophila) & $0.05 \pm 0.01$ \\
\hline
\end{tabular}

${ }^{\mathrm{a}}$ Fold-changes were shown in reference to B-cells

(A) RT-PCR
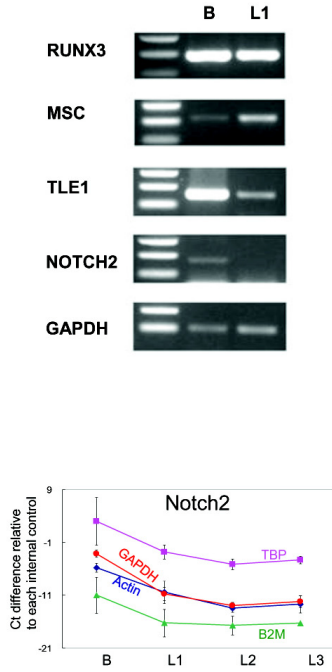

(C) Quantitative PCR using TBP normalizer

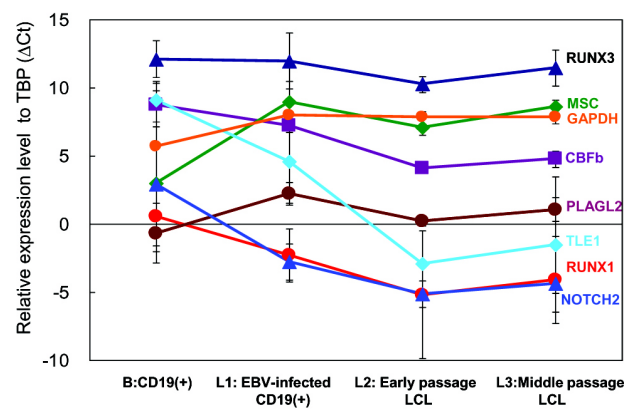

(B) Evaluation of internal controls
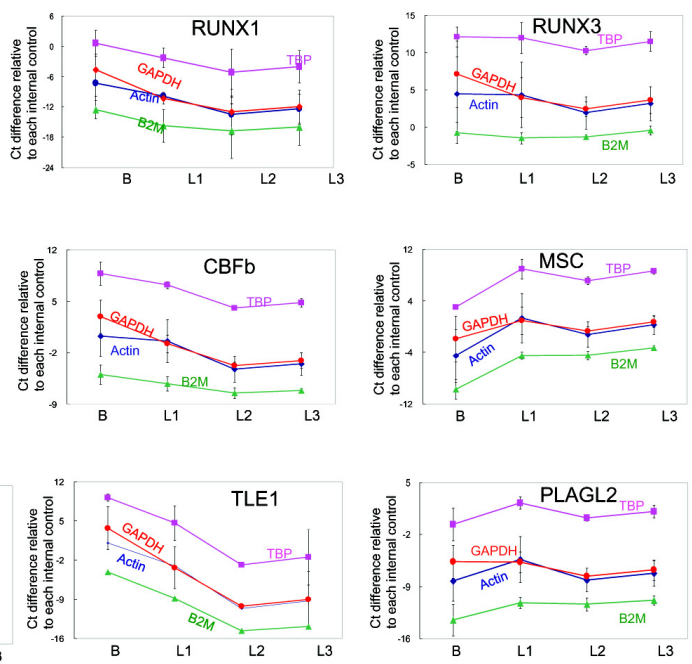
13

in

Fig. 1. Expression analysis of selected hematopoietic transcriptional regulatory genes using four common internal controls between B-cells and LCLs. Semi-quantitative RT-PCR was performed for some of target genes $(A)$, followed by quantitative real-time PCR of selected hematopoietic transcriptional regulatory genes using four common internal controls (B). Next, the relative expression levels were obtained using the $T B P$ internal control (C). B indicates Bcells, L1: LCLs at 5-8 weeks after EBV infection, L2 and L3: long-term subculture of LCLs at 50 and 100 passages, respectively. Relative expression levels, $\Delta\left(\mathrm{CT}_{\text {gene }}{ }^{-}\right.$

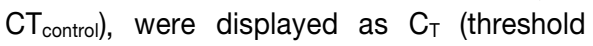
cycle) difference between genes of interest and internal control genes. Error bars indicate standard deviations of three experiments in duplicate. 
able internal control for gene expression analysis of various types of LCLs, we evaluated four common housekeeping genes as internal control genes. First, we extracted expression data on $A C T B, T B P, B 2 M$, and $G A P D H$ as well as some genes of interest from our previous microarray data (Baik et al., 2007), indicating upregulation of $G A P D H$ and $A C T B$ in LCLs compared to $\mathrm{B}$ cells. However, $T B P$ and $B 2 M$ exhibited less than a 2-fold change between B-cells and LCLs (Table 2). In addition, with regard to $B$ cell transformation, some genes of interest were randomly selected for expression studies of hematopoiesis and lymphomagenesis-related genes. Our microarray data showed that PLAGL2, MSC, TLE1, RUNX1, and NOTCH2 exhibited significant changes in gene expression levels between B-cells and LCLs (Table 2), whereas the other genes (i.e., RUNX3, $C B F B$ ) exhibited less than a 2-fold change. For these selected genes, RT-PCR had similar gene expression patterns for RUNX3, MSC, TLE1, and NOTCH2 as the microarray data (Fig. 1).

Four common housekeeping genes were included in the quantitative real-time PCR of hematopoietic transcriptional regulatory genes so that relative gene expression levels could be obtained by normalization. In a comparison of gene expression only between $B$ cells and early passages of LCLs, similar patterns in hematopoietic gene expression were observed when normalized by TBP, $A C T B$, and $B 2 M$ but not by $G A P D H$ (Fig. $1 \mathrm{~B})$, indicating that the expression pattern of $G A P D H$ deviated from those of the other housekeeping genes. This result suggested that GAPDH expression might be upregulated in EBV-infected B cells during LCL generation. Indeed, GAPDH upregulation was seen in LCLs of different passage numbers compared with $B$ cells when normalized by TBP (Fig. 1C).

Next, expression levels of hematopoietic genes were plotted in reference to each internal control (Fig. 1C). In a comparison of expression patterns between B cells and three different time points of long-term subcultures of LCLs (i.e., L1: EBV-infected B cells, L2: early passage LCLs, L3: late passage LCLs), correlation coefficients of individual expression patterns were calculated by pairwise comparison of normalized expression levels using four different internal controls (Table 3 ). For seven genes that were tested, TBP-normalized expression levels of each gene were correlated with $A C T B$-normalized expression levels, whereas the worst correlation coefficients of normalization between GAPDH and B2M were seen. This result suggested that $T B P$ or $A C T B$ might be a suitable internal control for gene expression analyses of hematopoiesis-related genes in various subculture passages of LCLs. However, our previous microarray data showed that $A C T B$ was upregulated by about 4fold in LCLs compared with B cells (Baik et al, 2007). Therefore, TBP was finally selected as an internal control for further gene expression analysis of hematopoietic transcriptional regulatory genes of interest.

Next, the optimized internal control, $T B P$, was applied to the gene expression analysis of seven randomly selected hematopoietic transcriptional regulatory genes in LCLs. Real-time PCR results showed that gene expression levels of MSC, TLE1, RUNX1, PLAGL2, and $G A P D H$ changed dramatically between $B$ cells and LCLs, whereas those of RUNX3 and $C B F B$ changed slightly after EBV infection (Fig. 1C). Interestingly, the upregulation or downregulation patterns that were detected in EBV-infected cells were sustained until the middle passage number (100) of LCLs. Thus, these results suggest that dysregulation of these selected hematopoietic genes may contribute to EBV-mediated B cell transformation and LCL immortalization.

\section{Discussion}

Gene expression studies of EBV-transformed LCLs have commonly used GAPDH or ACTB as internal controls for normalization (Baran-Marszak et al., 2002). In our previous study, global gene expression patterns between $B$

Table 3. Pairwise comparison of correlation coefficients of gene expression patterns.

\begin{tabular}{|c|c|c|c|c|c|c|}
\hline \multicolumn{7}{|c|}{ Correlation coefficients $^{a}$} \\
\hline Gene name & ACTB vs TBP & ACTB vs B2M & ACTB vs GAPDH & TBP vs B2M & TBP vs GAPDH & B2M vs GAPDH \\
\hline$R U N X 1$ & 0.995 & 0.928 & 0.963 & 0.959 & 0.983 & 0.992 \\
\hline$R \cup N X 3$ & 0.979 & 0.102 & 0.788 & 0.281 & 0.778 & 0.379 \\
\hline$C B F B$ & 0.985 & 0.938 & 0.920 & 0.984 & 0.974 & 0.998 \\
\hline PLAGL2 & 0.952 & 0.529 & 0.406 & 0.763 & 0.130 & -0.514 \\
\hline$M S C$ & 0.988 & 0.903 & 0.975 & 0.958 & 0.956 & 0.856 \\
\hline NOTCH2 & 0.993 & 0.948 & 0.983 & 0.979 & 0.998 & 0.990 \\
\hline TLE1 & 1.000 & 0.995 & 0.987 & 0.997 & 0.991 & 0.998 \\
\hline $\mathrm{AVE}^{\mathrm{b}}$ & 0.985 & 0.763 & 0.860 & 0.846 & 0.830 & 0.671 \\
\hline
\end{tabular}

${ }^{a}$ Correlation coefficients of the expression patterns of individual genes using normalization controls; ${ }^{b}$ average correlation coefficients. 
cells and LCLs were obtained from our microarray analysis, in which $G A P D H$ was used as an internal control for validation of gene expression (Baik et al., 2007). In general, internal control genes need relatively constant basal levels of gene expression in experimental conditions. However, our microarray data suggested that some housekeeping genes might be dysregulated between B cells and LCLs. Therefore, we evaluated four common housekeeping genes as suitable internal controls for the normalization of mRNA expression and applied them to a gene expression study of potential cellular target genes of EBV infection. Our results showed that the transcriptional level of $G A P D H$ increased in the process of EBV-mediated B-cell transformation. The upregulation of GAPDH might be involved in B-cell reprogramming during $L C L$ generation. Alternatively, GAPDH might be a direct cellular target gene of EBV infection. Generally, the expression of B cell-specific genes is lost, and $B$ lineage-inappropriate genes are upregulated during malignant transformation of lymphoid cells, suggesting B-cell reprogramming during EBV-mediated B-cell transformation (Janz et al., 2006). Therefore, GAPDH may not be suitable for internal controls in gene expression analyses between B-cells and LCLs. Furthermore, a correlation coefficient analysis for gene expression profiles, normalized by four housekeeping genes, showed that TBP was found to be a suitable internal control in the gene expression analysis between $B$ cells and LCLs. Moreover, TBP expression level was found to be moderately abundant in B cells and LCLs, as determined by the $\mathrm{C}_{T}$ difference between genes of interest and TBP (Fig. 1B). Although many other housekeeping genes were not evaluated in this study, at least TBP can be used as an optimum internal control for gene expression analysis, regardless of the status of B-cells (e.g., primary, EBV-infected B-cells) or long-term subculture of LCLs. Thus, we finally selected $T B P$ as a normalizer for further gene expression studies of hematopoietic transcriptional regulatory genes.

It is known that the hematopoietic genes that were selected for this study are involved in hematopoiesis and lymphoid malignancies. For example, PLAGL2, a developmentally regulated $\mathrm{C} 2 \mathrm{H} 2$ zinc finger on chromosome $8 \mathrm{q} 12$, is the main target for pleomorphic adenomas of the salivary gland. Recently, it was reported that PLAGL2 induced acute myeloid leukemia in cooperation with CBFB-MYH11 in mice (Landrette et al., 2005). Our results showed that PLAGL2 was upregulated in EBV-infected B-cells and LCLs, suggesting that PLAGL2 may be a potential target of EBV-mediated B-cell transformation. On the other hand, RUNX family members are heterodimeric transcription factors composed of an $\alpha$ subunit, RUNX, and a $\beta$ subunit, $\operatorname{CBF} \beta$. Of the three members of the RUNX family, RUNX1 is known as a master regulatory gene of hematopoiesis. According to the literature, RUNX3 was identified as a cellular target of $E B N A-2$ in the inducible cell line (Spender et al., 2002). The distal P1 promoter of RUNX1 controls expression of the RUNX1C isoform, while the proximal P2 promoter expresses RUNX1a $(A M L-1 a)$ and RUNX1b (AML-1b) (Miyoshi et al., 1991). RUNX3 represses transcription from the RUNX1 P1 promoter, indicating transcriptional crossregulation of RUNX1 by RUNX3 in human B cells (Spender et al., 2005). On the contrary, our PCR results showed that $R U N X 1$ expression was downregulated in parallel with slight downregulation of RUNX3 between $B$ cells and LCLs, which is inconsistent with a previous report of crossregulation by RUNX3. This discrepancy might be due to a different PCR target of $R U N X 1$ isoforms, because the RUNX isoform that we examined in our study was the RUNX1 P2 transcript rather than $R U N X 1 \mathrm{P} 1$ transcripts. Musculin (activated B-cell factor-1, MSO is a basic helix-loop-helix transcription factor, and its expression is restricted to a subset of lymphoid tissues, lymphoblastoid cell lines, and activated human B cells. MSC (ABF- 1$)$ expression is restricted to a subset of lymphoid tissues, lymphoblastoid cell lines, and activated human B cells (Massari et al., 1998), suggesting that MSC is a downstream target of the B-cell receptor signal transduction pathway. With respect to cell growth, EBV infection mimics B-cell activation signals or factors, such as CD40-CD40L, IgM, and LPS, so that EBV-infected B-cells exhibit greater proliferation than resting B-cells (Kilger et al., 1998). Thus, the upregulation of MSC in LCLs might be responsible for greater proliferative activity in EBV-infected B-cells compared with B-cells. TLE (transducin-like enhancer of split) genes are human homologs of the Drosophila groucho gene. TLE1 binds to the Runt domain and the $\mathrm{C}$ terminus of $R U N X 1$ to inhibit $R U N X 1$-induced transactivation of the CSF1 (colony stimulating factor 1) receptor. Our PCR analysis showed that TLE1 and NOTCH2 were downregulated in LCLs compared with B-cells, suggesting that Notch signaling may be involved in EBV-mediated B-cell transformation. Taken together, our results suggest that the hematopoietic transcriptional regulatory genes that were tested in this study may be potential cellular targets of EBV infection during LCL generation.

In conclusion, we evaluated four common internal controls for a gene expression analysis between $B$ cells and LCLs and found TBP as a suitable internal control. Expression analysis using a TBP normalizer allowed us to identify dramatic changes in selected hematopoietic transcriptional regulatory genes (downregulation of RUNX1, RUNX3, CBFB, TLE1, and NOTCH2; upregula- 
tion of MSC and PLAGL2) during EBV-mediated B-cell transformation and LCLs at different passages. Therefore, these genes may be potential cellular targets of EBV infection and potential reference biomarkers of LCL generation.

\section{Acknowledgements}

This work was supported by an intramural grant (2010N74001-00) of Korea National Institute of Health, Korea Centers for Disease Control and Prevention.

\section{References}

Baik, S.Y., Yun, H.S., Lee, H.J., Lee, M.H., Jung, S.E., Kim, J.W., Jeon, J.P., Shin, Y.K., Rhee, H.S., Kimm, K.C., and Han, B.G. (2007). Identification of stathmin 1 expression induced by Epstein-Barr virus in human B lymphocytes. Cell Prolif. 40, 268-281.

Baran-Marszak, F., Fagard, R., Girard, B., Camilleri-Broet, S., Zeng, F., Lenoir, G.M., Raphael, M., and Feuillard, J. (2002). Gene array identification of Epstein Barr virus-regulated cellular genes in EBV-converted Burkitt lymphoma cell lines. Lab. Invest. 82, 1463-1479.

Cahir-McFarland, E.D., Carter, K., Rosenwald, A., Giltnane, J.M., Henrickson, S.E., Staudt, L.M., and Kieff, E. (2004). Role of NF-kappa B in cell survival and transcription of latent membrane protein 1-expressing or Epstein-Barr virus latency III-infected cells. J. Virol. 78, 4108-4119.

Carter, K.L., Cahir-McFarland, E., and Kieff, E. (2002). Epstein-barr virus-induced changes in B-lymphocyte gene expression. J. Virol. 76, 10427-10436.

de Kok, J.B., Roelofs, R.W., Giesendorf, B.A., Pennings, J.L., Waas, E.T., Feuth, T., Swinkels, D.W., and Span, P.N. (2005). Normalization of gene expression measurements in tumor tissues: comparison of 13 endogenous control genes. Lab. Invest. 85, 154-159.

Filby, A.L., and Tyler, C.R. (2007). Appropriate 'housekeeping' genes for use in expression profiling the effects of environmental estrogens in fish. BMC Mol. Biol. 8, 10.

Haas, C.S., Creighton, C.J., Pi, X., Maine, I., Koch, A.E., Haines, G.K., Ling, S., Chinnaiyan, A.M., and Holoshitz, J. (2006). Identification of genes modulated in rheumatoid arthritis using complementary DNA microarray analysis of lymphoblastoid B cell lines from disease-discordant monozygotic twins. Arthritis Rheum. 54, 2047-2060.

Hur, D.Y., Lee, M.H., Kim, J.W., Kim, J.H., Shin, Y.K., Rho, J.K., Kwack, K.B., Lee, W.J., and Han, B.G. (2005). CD19 signalling improves the Epstein-Barr virus-induced immortalization of human B cell. Cell Prolif. 38, 35-45.

Janssens, N., Janicot, M., Perera, and T., Bakker, A. (2004). Housekeeping genes as internal standards in cancer research. Mol. Diagnostics Diagn. 8, 107-113.

Janz, M., Dorken, B., and Mathas, S. (2006). Reprogramming of B lymphoid cells in human lymphoma pathogenesis. Cell Cycle 5, 1057-1061.

Kang, M.S., Lu, H., Yasui, T., Sharpe, A., Warren, H.,
Cahir-McFarland, E., Bronson, R., Hung, S.C., and Kieff, E. (2005). Epstein-Barr virus nuclear antigen 1 does not induce lymphoma in transgenic FVB mice. Proc. Natl. Acad. Sci. U.S.A. 102, 820-825.

Kelly, G., Bell, A., and Rickinson, A. (2002). Epstein-Barr virus-associated Burkitt lymphomagenesis selects for downregulation of the nuclear antigen EBNA2. Nat. Med. 8, 1098-1104.

Kilger, E., Kieser, A., and Baumann, M. (1998). Hammerschmidt $W$. Epstein-Barr virus-mediated B-cell proliferation is dependent upon latent membrane protein 1 , which simulates an activated CD40 receptor. EMBO J. 17, 17001709.

Landrette, S.F., Kuo, Y.H., Hensen, K., Barjesteh van Waalwijk., van Doorn-Khosrovani, S., Perrat, P.N., Van de Ven, W.J., Delwel, R., and Castilla, L.H. (2005). Plag1 and PLAGL2are oncogenes that induce acute myeloid leukemia in cooperation with CBFB-MYH11. Blood 105, 2900-2907.

Lossos, I.S., Czerwinski, D.K., Wechser, M.A., and Levy, R. (2003). Optimization of quantitative real-time RT-PCR parameters for the study of lymphoid malignancies. Leukemia 17, 789-795.

Massari, M.E., Rivera, R.R., Voland, J.R., Quong, M.W., Breit, T.M., van Dongen, J.J., de Smit, O., and Murre, C. (1998). Characterization of ABF-1, a novel basic helix-loop-helix transcription factor expressed in activated $B$ lymphocytes. Mol. Cell. Biol. 18, 3130-3139.

Miyoshi, H., Shimizu, K., Kozu, T., Maseki, N., Kaneko, Y., and Ohki, M. (1991). t(8;21) breakpoints on chromosome 21 in acute myeloid leukemia are clustered within a limited region of a single gene, AML1. Proc. Natl. Acad. Sci. U.S.A. 88, 10431-10434.

Natkunam, Y., Lossos, I.S., Taidi, B., Zhao, S., Lu, X., Ding, F., Hammer, A.S., Marafioti, T., Byrne, G.E. Jr. Levy, S., Warnke, R.A., and Levy, R. (2005). Expression of the human germinal center-associated lymphoma (HGAL) protein, a new marker of germinal center B-cell derivation. Blood 105, 3979-3986.

Ohl, F., Jung, M., Xu, C., Stephan, C., Rabien, A., Burkhardt, M., Nitsche, A., Kristiansen, G., Loening, S.A., Radonić, A., and Jung, K. (2005). Gene expression studies in prostate cancer tissue: which reference gene should be selected for normalization? J. Mol. Med. 83, 1014-1024.

Rho, H.W., Lee, B.C., Choi, E.S., Choi, I.J., Lee, Y.S., and Goh, S.H. (2010) Identification of valid reference genes for gene expression studies of human stomach cancer by reverse transcription-qPCR. BMC Cancer 10, 240-252.

Saunders, C.I., Kunde, D.A., Crawford, A., and Geraghty, D.P. (2007). Expression of transient receptor potential vanilloid 1 (TRPV1) and 2 (TRPV2) in human peripheral blood. Mol. Immunol. 44, 1429-1435.

Selvey, S., Thompson, E.W., Matthaei, K., Lea, R.A., Irving, M.G., and Griffiths, L.R. (2001). Beta-actin--an unsuitable internal control for RT-PCR. Mol. Cell. Probes 15, 307311.

Shukla, S.J., and Dolan, M.E. (2005). Use of CEPH and non-CEPH lymphoblast cell lines in pharmacogenetic 
studies. Pharmacogenomics 6, 303-310.

Simon-Sanchez, J., Scholz, S., Fung, H.C., Matarin, M., Hernandez, D., Gibbs, J.R., Britton, A., de Vrieze, F.W., Peckham, E., Gwinn-Hardy, K., Crawley, A., Keen, J.C., Nash, J., Borgaonkar, D., Hardy, J., and Singleton, A. (2007). Genome-wide SNP assay reveals structural genomic variation, extended homozygosity and cell-line induced alterations in normal individuals. Hum. Mol. Genet. $16,1-14$

Spender, L.C., Cornish, G.H., Sullivan, A., and Farrell, P.J. (2002). Expression of transcription factor AML-2 (RUNX3, $\mathrm{CBF}($ alpha)-3) is induced by Epstein-Barr virus EBNA-2 and correlates with the B-cell activation phenotype. J. Virol. 76, 4919-4927.

Spender, L.C., Whiteman, H.J., Karstegl, C.E., and Farrell, P.J. (2005). Transcriptional cross-regulation of $R U N X 1$ by RUNX3 in human B cells. Oncogene 24, 1873-1881.

Stranger, B.E., Forrest, M.S., Dunning, M., Ingle, C.E., Beazley, C., Thorne, N., Redon, R., Bird, C.P., de Grassi,
A., Lee, C., Tyler-Smith, C., Carter, N., Scherer, S.W., Tavare, S., Deloukas, P., Hurles, M.E., and Dermitzakis, E.T. (2007). Relative impact of nucleotide and copy number variation on gene expression phenotypes. Science 315, 848-853.

Theis, T., Skurray, R.A., and Brown, M.H. (2007). Identification of suitable internal controls to study expression of a Staphylococcus aureus multidrug resistance system by quantitative real-time PCR. J. Microbio. Methods 70, 355-362.

Thellin, O., Zorzi, W., Lakaye, B., De Borman, B., Coumans, B., Hennen, G., Grisar, T., Igout, $A_{\text {., }}$ and Heinen, E. (1999). Housekeeping genes as internal standards: use and limits. J. Biotechnol. 7, 291-295.

Vandesompele, J., De Preter, K., Pattyn, F., Poppe, B., Van Roy, N., De Paepe, A., and Speleman, F. (2002). Accurate normalization of real-time quantitative RT-PCR data by geometric averaging of multiple internal control genes. Genome Biol. 3, research 0034.1-0034.11 